\title{
Visual cues effect on the impact of sonification on movement
}

\author{
Joseph W Newbold \\ UCL Interaction Centre \\ UCL London, London \\ joseph.newbold.14@ucl.ac.uk
}

\section{Nicolas E Gold}

Dept of computer science

UCL London, London

n.gold@ucl.ac.uk

\author{
Nadia Bianchi-Berthouze \\ UCL Interaction Centre \\ UCL London, London \\ n.berthouze@ucl.ac.uk
}

\section{ABSTRACT}

When designing movement sonification, its effect on peoples' movement must be considered. Recent work has shown that using musical expectancy within movement sonification can impact the way people move. However, this body of work has also found that different types of movements show different effects of these musically-informed sonifications on movement behaviour. This paper addresses this disparity, by exploring how this kind of sonification may be impacted by perceptual cues. We focus on two kinds of target movements: an "open movement", i.e. one with limited perceptual cues (no visual cues from the environment and limited proprioceptive cues) at a target point, and a "closed movement" i.e. one with strong perceptual (visual and proprioceptive) cues at the target point. We present a study that investigates how visual cues may interact with musical expectancy based sonification and discuss what these results mean for musical expectancy based designs and musically-informed sonification overall We show that the effect of expectancy of sonification at a target point is greater when visual cues are removed. 


\section{CCS CONCEPTS}

- Human-centered computing $\rightarrow$ Auditory feedback;

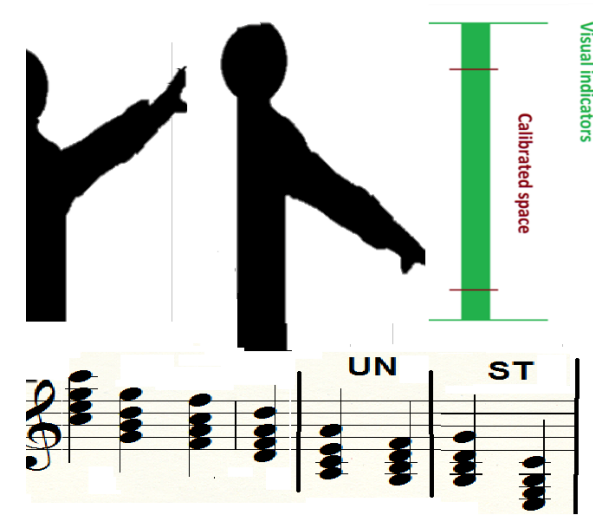

Figure 1: Depicting the experimental setup for the study, the movement is calibrated within the space and as the participant moves their arm they hear the given chord sequence ending in either a harmonically stable (ST) or unstable (UN) ending.

\section{KEYWORDS}

movement sonification; sonification design; movement behaviour; physical activity.

\section{ACM Reference Format:}

Joseph W Newbold, Nadia Bianchi-Berthouze, and Nicolas E Gold. 2018. Visual cues effect on the impact of sonification on movement. In Proceedings of MOCO '18 (MOCO '18'). ACM, New York, NY, USA, 6 pages. https://doi.org/xxxxx

\section{INTRODUCTION}

Movement sensing is increasing being used to provide real-time feedback to support physical activity, specifically movement sonification has been shown to be beneficial for supporting movement $[4,11,13]$ Sound can be used to guide or inform people of their movement and allow them to reflect on it with a new perspective. Recent work has begun to explore how sonification may unconsciously impact movement behaviour itself $[2,12]$ beyond simply guiding along a trajectory, aiming to design sonification techniques that subconsciously alter movement behaviour. Previous work has also found that the inclusion of high-level musical structures within sonification, such as melody, rhythm and harmony can make use of people's embodied understanding of music to alter the way people move $[4,7,8]$. However, the interaction of other external perceptual cues (e.g. visual feedback) with such sonifications has yet to be explored. In this paper, we examine how altering the level of visual cues available can lead to a change in the impact of musical expectancy on movement. Musical expectancy is defined by the feeling of how a given piece of music should continue (or complete), which we learn from our day to day exposure to music [1]. The results of this study allow us to better understand how musical expectancy can be used as a mapping parameter in sonifications to support physical activity and how different movement types should be considered in the design of movement sonifications.

\section{BACKGROUND}

\subsection{Use of Music in Sonification}

Movement sonification has been used to support motor-learning for new movements [4] and to help support movement $[11,13]$. Additionally, music has been shown to both provide motivation and change our movement [7,8], betraying the embodied relationship we have with music [6]. The use of musical structures has been used to augment movement sonification, specifically in this paper we will explore the use of structures from western tradition music. Wallis et al. designed a sonification which used harmonic resolution to reward the completion of the movement [13] and Singh et al. found a 
more melodic sonification was preferred and increased self-efficacy in chronic pain rehabilitation [11]. These works demonstrate how music can be a powerful tool for augmenting movement sonification. However, there has been little investigation into how musical structure within sonification may influence the way in which people move.

Recent work has begun to explore more explicitly how these musical structures may influence movement behaviour. Dyer et al. compare melodic versus rhythmic based sonifications and find that not only did the melodic sonification have greater immediate retention, but after 24 hours a replay of the melodic structure was able to improve the movement execution without the use of the sonification [4]. Newbold et al., show how the sonification of target points while stretching forward can use harmonic resolution to alter movement behaviour, mapping the completion of a stretch forward movement to either a musically complete or incomplete ending, they found that the sound could not only be used to motivate/reward the movement but also affect how people's movement changed, moving more when the ending sounded incomplete [8]. It can also been seen how sonifications without using musical structure have also been shown to alter both people's perceptions and movement behaviour[12][2]. These works show that sound-feedback impacts movement, however currently both musical and non-musical sound-based manipulation has considered the impact of other external perceptual cues

\subsection{Impact of Perceptual Cues}

People are primarily "visually dominant", i.e. they will rely more on visual input than other modalities [3]. Petrini et al. show that children will even use irrelevant visual cues to help localise sound, showing that learning to ignore these cues can be difficult [9]. These interactions must be considered when design sonifications, especially those that aim to alter people's behaviour, as an unexpected interaction between visual and auditory cues may lead to this kind of sonification being ineffective.

Considering the work by Newbold et al., the use of musical expectancy has been shown to impact movement behaviour at the target point of a stretch forward movement [8]. However, the same kind of sonification design for the squat down movement does not find the same impact [7]. The level of cues at the target point is very different for these two movements. Stretching forward there is no definite conceptual endpoint for the movement and a lack of visual cues, while when squatting there is a very definite endpoint as one approaches the ground and clear visual cues. These different levels of cues present and people's propensity to rely on visual cues may be what leads to a difference in the impact on movement behaviour.

\section{INVESTIGATING MUSICAL EXPECTANCY SONIFICATION AND VISUAL CUES}

To investigate the disparity between musical expectancy's effect on movement behaviour, a study in which the level of cues could be manipulated was designed. A vertical arm movement was chosen for 
- H1 There will be significant interaction between the level of cues and the stability

- $\mathbf{H 2}$ when cues are low people will move more and take longer in the unstable versus the stable

- H3 People will report decreased confidence and increased informativeness when cues are low

- H4 We will still see differences in motivation/reward for stable versus unstable conditions

Sidebar 1: For the study we have four main hypotheses the study to limit the tactile cues given at the extremes of a movement. For this, we use a sonification design as used in $[7,8]$ and measure the impact the sound has on movement with and without visual cues. We expect to see the visual cues impact the effectiveness of the expectancy, as participants rely on the visual cues they are less easily manipulated by the sound. Similarly, the visual markers will increase participants confidence as they use the visual feedback to confirm their position, conversely without the visuals the sound will seem more informative. Additionally, the impacts on motivation/reward will be unaffected by perceptual cues as seen in [7, 8], see Sidebar 1 .

\subsection{Participants \& Materials}

25 paid participants were recruited for the study (18 female and 7 male, mean age $=24.4(18-44)$. A smartphone orientation sensor (50FPS) was used to measure the movement for both sonification and analysis. The measurement used the angle of the straight arm as it moved through the space as seen in figure 1. The sonified movement space, that is the space in which the movement will be sonified, was calibrated to fall within the two visual markers: two lines on a whiteboard in front of participants. Therefore participants would use it more as a cue than as the target point itself. As the arm moves a set chord progression are played, ending in either a complete or incomplete cadence see fig 1. A set of discrete chords was used to better convey a specific musical key, which can then end in resolution (perfect cadence) or tension (imperfect cadence), additionally as there is space in which a chord would not sound, the impact of the cadence can be seen more clearly as the music is perceived as either complete/incomplete, as opposed to silence after the sound being used as the main identifier.

\subsection{Design \& Procedure}

A randomised within-subjects design was used for the 4 trials. There were 2 independent variables: harmonic stability (stable (ST) / unstable (UN)), Level of visual cues (high (HC) with the visual marker visible /low(LC where participants had their eyes closed)). For behavioural measures, the deviation past the target point and the time taken to reach the maximum extension were measured using the smartphone, from the amount of movement between the final chord (in degrees) and the time taken to do so (in frames). In terms of self-reported measures, perceived harmonic stability of the ending, perceived confidence they had reached the target, perceived informativeness of the sound, perceived motivation to continue past the target point and perceived amount of reward were all measured using a 7-point Likert scale. After an introduction and demonstration of the device, the smartphone was placed on the participant's arm. Participants were instructed to move their arm to the target point and back to their starting position for two repetitions using the music produced to guide them. Specifically, they were told when they felt they had reached the end of the chords they should return. 


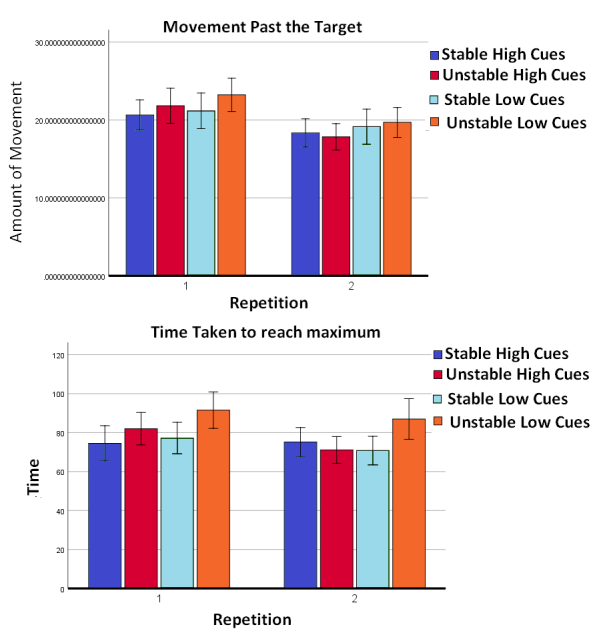

Figure 2: Mean (SE) for the behavioural measures, movement past the target in degrees and time taken, in frames, for each repetition.

\begin{tabular}{|l|l|l|l|l|}
\hline & & & & \\
& STHC & UNHC & STLC & UNLC \\
\hline Stability & $2(1-5)$ & $4(1-6)$ & $2(1-4)$ & $3(1-6)$ \\
\hline Confidence & $6(2-7)$ & $5(3-7)$ & $6(2-7)$ & $5(2-7)$ \\
\hline Informative & $5(2-7)$ & $4(2-7)$ & $6(2-7)$ & $6(3-7)$ \\
\hline Motivation & $3(1-7)$ & $2.5(1-7)$ & $2.5(1-6)$ & $2.5(1-7)$ \\
\hline Reward & $4(1-7)$ & $3(1-7)$ & $5(1-7)$ & $3(1-7)$ \\
\hline
\end{tabular}

Figure 3: Median(IQR) showing selfreport results. For Stable with high perceptual cues (STHC), unstable with high perceptual cues (UNHC), stable with low perceptual cues (STLC) and unstable with low perceptual cues (UNLC)

\subsection{Results}

Behavioural measures, normalised(square root), for the two repetitions were submitted to a repeated measures analyses of variance (ANOVA) with stability and target point as within-subject factors.

No significant effects were found for the amount of movement. For the first repetition, the impact of perceptual cues on the time taken to reach the maximum displacement of the arm was found to be significant $\left(F(1,24)=14.073 p=.001, \chi^{2}=.370\right)$. Participants took longer to reach the maximum point in the first repetition when they had limited visual perceptual cues. For the second repetition, no significant effect was found for musical resolution or target point, however there was a significant interaction $\left(F(1,24)=5.305 p=.030, \chi^{2}=.181\right)$. Paired tests (with an adjusted significance level $(\alpha=0.0125)$ are approaching significant for rep 2 between STLC and UNLC $(T(24)=-2.191, p=.038)$ and between UNLC and UNHC $(T(24)=-2.247, p=.034)$. Suggesting that when perceptual cues are low, there is a significant effect of stability on the time taken to reach maximum stretch. A series of Friedman tests found significant differences for: perceived stability $\left(\chi^{2}(3)=13.148, p=.004\right)$, confidence $\left(\chi^{2}(3)=11.190, p=.011\right)$ and perceived informativeness $\left(\chi^{2}(3)=8.594, p=.004\right)$. Subsequent Wilcoxon tests $(\alpha=0.0125)$ showed that STHC and STLC were more stable than UNHC $(Z=-2.765, p=.006)$ and $(Z=-3.070, p=.002)$. For confidence, there was a significant difference between STHC and UNLC $(Z=-2.670, p=.008)$ as well as for informativeness, $(Z=-2.946, p=.003)$.

\section{DISCUSSION}

The results of the testing of our hypotheses are summarised in sidebar 2 and while in the second repetition we do see a significant interaction in the time taken to complete the movement, we do not find that in the first rep, both endings lead to a longer movement time when cues were low, implying that without visual cues people move with more uncertainty, but in the second rep the stable conditions make people more certain, leading to shorter movement times, see figure 2 . From these results, we can see that, like the squat down movement, there is little impact on the amount of movement past the target point [7]. This could be related to each condition having the same number of notes, making it clearer when one had reached the target point. Additionally, it could be that the level of proprioception in the shoulder is too high to be impacted significantly; as shown by Hall and McCloskey proprioception at the shoulder is relatively high [5]. However, some impact is seen on the time taken, which correlates with both our reliance on visual cues and faster response times for musically complete stimulus $[3,10]$. While these results indicate there may be an impact of visual cues on sonification, further study is needed. Through exploring these cues, we can better understand in which situations these movement altering sonifications may be effective. 


\section{CONCLUSION}

This paper has explored how visual cues may affect the way in which sonifications may impact movement behaviour. In the presented study, while some impacts of the level of visual cues are seen, for the given movement there is limited change in the amount of movement past the target point. In conclusion, the work shows that the use of sonification may be dependent on movement type. The results demonstrate how the context of the movement must be considered when designing movement sonifications and the impact of perceptual cues must be explored when they aim to alter movement.

- H1 The significant interaction between level of cues and harmonic stability was found for the time taken, in the second rep only

- H2 Follow-up t-tests show a difference between stable and unstable endings when perceptual cues were low that was approaching significant

- H3 Participants were more confident they had reached the target point when perceptual cues where high and participants did found the sounds more informative when perceptual cues were low

- H4 Participants did not find any conditions significantly more or less motivating or rewarding, this is perhaps related to the trivialness of the movement itself not needing them to feel motivated or giving much sense of reward

Sidebar 2: How results compare to our four main hypotheses

\section{ACKNOWLEDGMENTS}

Supported by EPSRC EP/H017178/1 'Emo \& Pain' and by an EPSRC DTG.

\section{REFERENCES}

[1] Emmanuel Bigand. 1997. Perceiving musical stability: The effect of tonal structure, rhythm, and musical expertise. ...of Experimental Psychology: Human Perception and ... (1997). http://psycnet.apa.org/journals/xhp/23/3/808/

[2] Eric Olivier Boyer, Bénédicte Maria Babayan, Frédéric Bevilacqua, Markus Noisternig, Olivier Warusfel, Agnes Roby-Brami, Sylvain Hanneton, and Isabelle Viaud-Delmon. 2013. From ear to hand: the role of the auditory-motor loop in pointing to an auditory source. Frontiers in computational neuroscience 7 (2013), 26.

[3] Francis B. Colavita. 1974. Human sensory dominance. Perception \& Psychophysics 16, 2 (01 Mar 1974).

[4] J. F. Dyer, P. Stapleton, and M. W. M. Rodger. 2017. Advantages of melodic over rhythmic movement sonification in bimanual motor skill learning. Experimental Brain Research 235, 10 (01 Oct 2017).

[5] L A Hall and D I McCloskey. [n. d.]. Detections of movements imposed on finger, elbow and shoulder joints. The Journal of Physiology 335, 1 ([n. d.]).

[6] Marc Leman. 2008. Embodied music cognition and mediation technology. Mit Press.

[7] Joseph W Newbold, Nadia Bianchi-Berthouze, and Nicolas E Gold. 2017. Musical Expectancy in Squat Sonification for People who Struggle with Physical Activity. In International Conference on Auditory Display. Penn State University.

[8] Joseph W. Newbold, Nadia Bianchi-Berthouze, Nicolas E. Gold, Ana Tajadura-Jiménez, and Amanda CdC Williams. 2016. Musically Informed Sonification for Chronic Pain Rehabilitation: Facilitating Progress \& Avoiding Over-Doing. In CHI '16.

[9] Karin Petrini, Peter R. Jones, Louise Smith, and Marko Nardini. 2015. Hearing Where the Eyes See: Children Use an Irrelevant Visual Cue When Localizing Sounds. Child Development 86, 5 (2015). https://doi.org/10.1111/cdev.12397

[10] E Glenn Schellenberg, Emmanuel Bigand, Benedicte Poulin-Charronnat, Cécilia Garnier, and Catherine Stevens. 2005. Children's implicit knowledge of harmony in Western music. Developmental science 8, 6 (Nov. 2005).

111] Aneesha Singh, Annina Klapper, Jinni Jia, Antonio Fidalgo, Ana Tajadura-Jiménez, Natalie Kanakam, Nadia BianchiBerthouze, and Amanda Williams. 2014. Motivating People with Chronic Pain to Do Physical Activity: Opportunities for Technology Design. In CHI '14. ACM, New York, NY, USA, 2803-2812.

[12] Ana Tajadura-Jiménez, Maria Basia, Ophelia Deroy, Merle Fairhurst, Nicolai Marquardt, and Nadia Bianchi-Berthouze. 2015. As light as your footsteps: altering walking sounds to change perceived body weight, emotional state and gait. (2015).

[13] Richard Isaac Wallis, Todd Ingalls, Thanassis Rikakis, Loren Olsen, Yinpeng Chen, Weiwei Xu, and Hari Sundaram. 2007. Real-time sonification movement for an immersive stroke rehabilitation environment. In (ICAD 2007). 